\title{
Detailed In Situ Observations of Electromigration in Aluminum Wires
}

\author{
Matthew Mecklenburg ${ }^{1}$, Brian Zutter ${ }^{2}$, William A. Hubbard ${ }^{2}$, Shaul Aloni ${ }^{3}$, and B. C. Regan ${ }^{2}$ \\ ${ }^{1 .}$ Center for Electron Microscopy and Microanalysis, University of Southern California, Los Angeles, \\ CA, USA \\ 2. Department of Physics and Astronomy \& California NanoSystems Institute, University of California, \\ Los Angeles, CA, USA \\ 3. Molecular Foundry, Lawrence Berkeley National Laboratory, Berkeley, CA, USA
}

Atoms in a current-carrying metal wire can experience electromigration, where the electric field and associated electric current drive atomic diffusion [1]. Understanding this process is important for the successful very-large-scale integration (VLSI) of integrated circuits (ICs) [2], since each IC contains many small wires subject to large current densities. Using scanning transmission electron microscopy (STEM) and electron energy loss spectroscopy (EELS), we have observed in situ electromigration in aluminum wires suspended on electron-transparent, $15 \mathrm{~nm}$-thick silicon nitride membranes. In some cases, a void that forms with one sign of the electrical current will be subsequently filled when the current is reversed, indicating the existence of sites that are particularly disposed to both sourcing and sinking atoms (see Figure 1).

The ability to observe with in situ TEM such gross electromigration-induced changes has been available for many years [3]. However, using recently developed techniques [4] based on valence EELS, it is now possible to efficiently determine changes in the structure of a metal wire that are much more subtle. By mapping the bulk plasmon energy, we can quantify density changes throughout an aluminum wire, both while it carries electric current and after the current has been switched off. Some of the density changes are transient; these are present only when the current is on, and indicate the presence of forces below the threshold required to move atoms from one lattice site to another. Other density changes persist in the absence of any current; these indicate that electromigration has occurred, with relocation of atoms that is generally, at least in part, irreversible.

Figure 1 contains three pair of images showing respectively an aluminum wire, the same wire after it has carried first $2 \mathrm{~mA}$ to the right for 30 minutes, and then $2 \mathrm{~mA}$ to the left for 30 minutes (a current density of $1.3 \times 10^{7} \mathrm{~A} / \mathrm{cm}^{2}$ in each direction). The wire is $100 \mathrm{~nm}$ thick, $150 \mathrm{~nm}$ wide, and $3 \mu \mathrm{m}$ long. No current was flowing in the wire as these images were acquired. The first image in each pair (i.e. A, C, and E), the standard annular dark field STEM image, shows gross structural modifications. For instance, a void near the center of the wire expands between $\mathrm{A}$ and $\mathrm{C}$, and then refills between $\mathrm{C}$ and $\mathrm{E}$.

The second image in each pair (i.e. B, D, and F), a map of the plasmon energy, reveals a wealth of additional detail. Not only are the growth and shrinkage of voids evident, but the grain boundary structure of the wire is much easier to see [4]. Here the typical size of the grains closer to the contacts, where the wire heats less, is smaller than it is nearer the center of the wire. A large grain boundary happens to bisect the wire near its midpoint. Interestingly, the plasmon energy to the left of this boundary increases in F relative to B and D, indicating that not only has the void of D been filled, the local atomic density has been increased relative to that of image B. Further measurements of transient and persistent electromigration effects via on quantitative plasmon energy mapping will be presented at the meeting. 


\section{References:}

[1] DG Pierce and PG Brusius, Microelectronics Reliability 37 (1997), p. 1053-1072.

[2] KN Tu, Journal of Applied Physics 94 (2003), p. 5451.

[3] BC Regan et al, Nature 428 (2004), p. 924-927.

[4] M Mecklenburg et al, Science 347 (2015), p. 629-632.

[5] This work was supported by FAME, one of six centers of STARnet, a Semiconductor Research Corporation program sponsored by MARCO and DARPA, by National Science Foundation (NSF) award DMR-1611036, and by NSF STC award DMR-1548924. Data presented were acquired at the Center for Electron Microscopy and Microanalysis at the University of Southern California. D. R. G. Mitchell's "Apply CLUT" script was used to make the intensity scale bar in Figure 1.

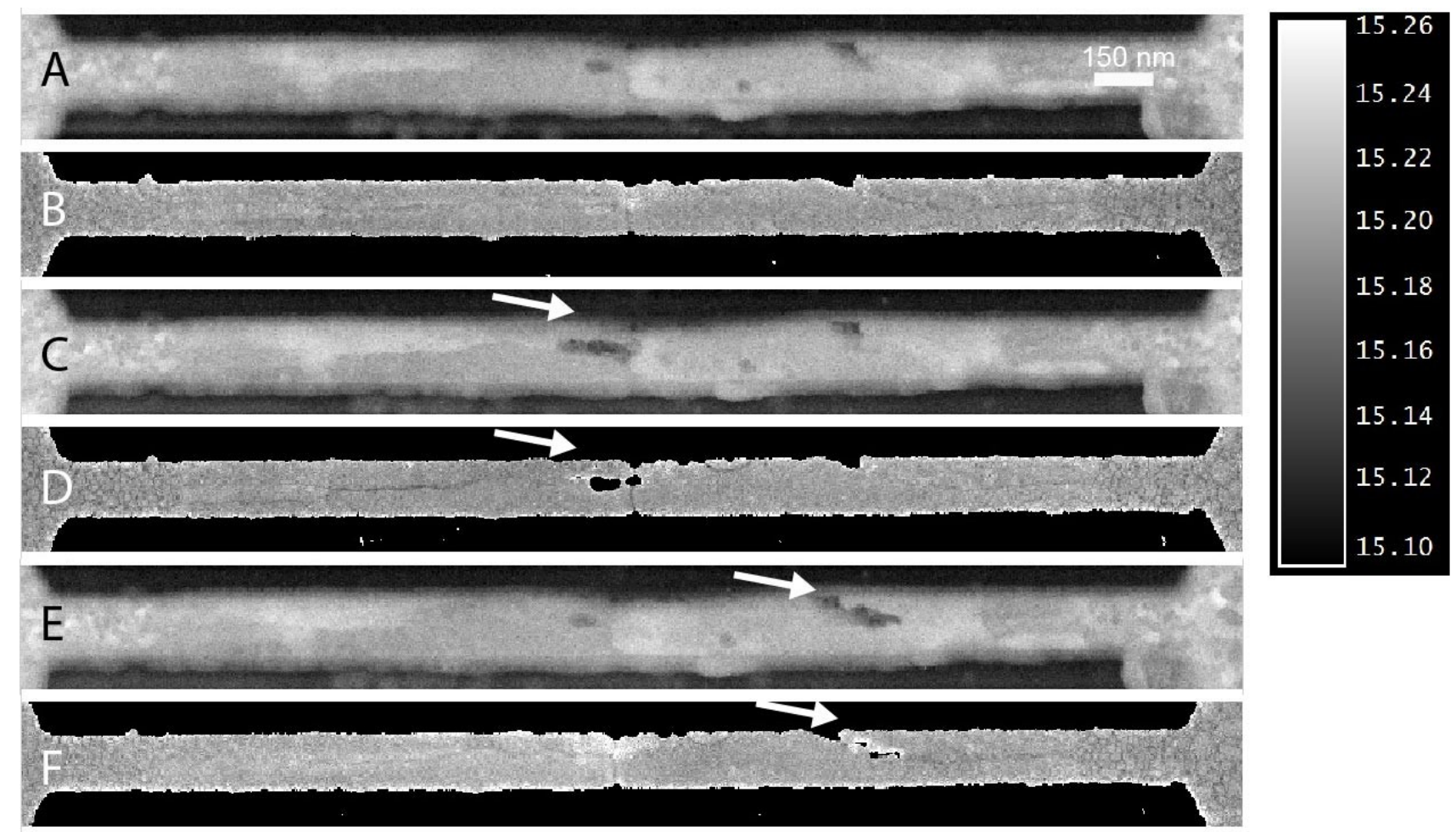

Figure 1. Annular dark-field STEM images (A, C and E) and plasmon energy maps (B, D, and F) of an aluminum wire. The three pairs show the wire, respectively, in an initial (though not pristine) condition, after current has passed through the wire left to right (positive voltage on the left), and after current has passed through the wire in the opposite direction. An intensity scale bar for the plasmon energy maps is shown at right. Arrows point to examples of voids formed after each application of current. 